\title{
Einflüsse auf die elektroosmotische Durchströmung von nanoporösen Haufwerken
}

\section{Bastian Schäfer *, Hermann Nirschl}

Die Durchströmung von Haufwerken, in denen Partikel in engem Kontakt mit ihren Nachbarn stehen, ist für viele industrielle Prozesse von Bedeutung. In nanoporösen Haufwerken sind Stoff- und Ladungstransport miteinander verknüpft. Diese elektrokinetischen Effekte bieten die Möglichkeit, das Problem des hohen Durchströmungswiderstandes zu umgehen. Der hohe Durchströmungswiderstand schränkt den Einsatz von Nanopartikeln ein, z. B. als Katalysatorträger in Festbettreaktoren. Die hier untersuchten Haufwerke wurden durch Filtration von wässrigen Titandioxid- und Aluminiumoxidsuspensionen hergestellt und anschließend mit Elektrolytlösungen verschiedener $\mathrm{pH}$-Werte und Ionenkonzentrationen durchströmt. Die druckgetriebene Durchströmung führt zur Ausbildung eines Strömungspotenzials. Das Anlegen einer externen Spannung ruft hingegen einen elektrischen Strom und einen elektroosmotischen Volumenstrom hervor. Die Messergebnisse zeigen eine klare Abhängigkeit des Strömungspotenzials und des elektroosmotischen Volumenstroms vom pH-Wert und der Ionenstärke der Elektrolytlösung.

\section{Einleitung}

Die Durchströmung von Haufwerken mit Elektrolytlösungen ist für viele industrielle Prozesse relevant. Beispielhaft sei hierfür die Durchströmung von Filterkuchen und Festbettreaktoren angeführt. Anwendungsmöglichkeiten für Haufwerke, die aus nanoskaligen Partikeln und Poren bestehen, im Folgenden als nanoporöse Haufwerke bezeichnet, sind z. B. in der Separationstechnik, in der Verkleinerung von Katalysatoren oder in der Miniaturisierung von elektroosmotischen Pumpen zu finden. Für die Realisierung dieser Anwendungen ist es wichtig, den hohen Durchströmungswiderstand von nanoporösen Haufwerken $\mathrm{zu}$ verstehen und $\mathrm{zu}$ minimieren. Die etablierten Modelle für die Durchströmung von makroskaligen Haufwerken lassen sich nicht auf nanoporöse Haufwerke übertragen, da die Modellannahmen wegen der zusätzlich auftretenden Grenzflächenkräfte nicht gelten. Diese Grenzflächenkräfte beruhen auf der Ausbildung von elektrochemischen Doppelschichten auf den Partikeloberflächen. Dabei bilden spezifisch adsorbierte Ionen die innere Helmholtz-Schicht. Die entgegengesetzt geladenen Gegenionen der Elektrolytlösung werden angezogen und ordnen sich in der starren äußeren Helmholtz-Schicht an. Um diese herum bildet sich der diffuse Teil der Doppelschicht aus, eine deformierbare „Ionenwolke“ mit exponentiellem Abklingen der Ionenkon- zentration und des elektrischen Potenzials. Die Abklinglänge wird als Debye-Länge bezeichnet.

Die Adsorption von Ionen an der Oberfläche der Partikel hängt von deren Material und von der ionischen Zusammensetzung der Flüssigkeit ab. Bei niedrigen $\mathrm{pH}$-Werten, also bei hoher $\mathrm{H}_{3} \mathrm{O}^{+}$-Konzentration, tragen die Partikel eine positive Ladung. Bei hohen $\mathrm{pH}$-Werten bilden sich negative Partikelladungen aus. Dazwischen befindet sich der materialspezifische isoelektrische Punkt (IEP), bei dem die Partikel keine Ladungen tragen. Da die Messung der Oberflächenladung sehr aufwändig ist, dient meist das Zeta-Potenzial als Referenz. Das Zeta-Potenzial ist die Potenzialdifferenz zwischen der Flüssigkeit und der Scherebene, die sich bei einer relativen Bewegung zwischen Partikel und Flüssigkeit ausbildet. Dabei wird die diffuse Doppelschicht zum größten Teil abgeschert [1].

Die elektrochemische Doppelschicht beeinflusst die Durchströmung von Haufwerken, deren Partikel in einer Größenordnung von Mikrometern oder Nanometern liegen. Bei makroskaligen Partikeln spielen diese Grenzflächeneffekte wegen der kleinen spezifischen Oberfläche eine untergeordnete Rolle. Tab. 1 zeigt eine Klassifizierung der Kräfte, die auf Partikel in einem durchströmten Haufwerk wirken.

- In makroskaligen Haufwerken beschränken sich die interpartikulären Wechselwirkun-

Die Durchströmung
von Haufwerken
mit Elektrolyt-
lösungen ist für
viele industrielle
Prozesse relevant.
Beispiel hierfür ist
die Durchströmung
von Filterkuchen
und Festbett-
reaktoren.
reaktoren. 


\section{Bei stark geladenen Partikeln verhindert die elektrostatische Abstoßung die Agglomeration der Partikel. Ungela- dene Partikel bilden hingegen große Agglomerate aus.}

gen auf die Kontaktkräfte zwischen benachbarten Partikeln. Diese Kontaktkräfte resultieren aus der Kompression und aus dem Durchströmungswiderstand des Haufwerkes. In nanoporösen Haufwerken wirken zwischen den Partikeln die elektrostatische Abstoßung aufgrund der Coulomb-Kraft, die Born'sche Abstoßung und die Anziehung aufgrund der van der Waals-Kraft. Das Gleichgewicht der Kräfte lässt sich mit der DLVO-Theorie beschreiben [1] und bestimmt die Struktur eines Haufwerkes, das durch Filtration einer nanopartikulären Suspension gebildet wurde (s. Abb. 1). Bei stark geladenen Partikeln verhindert die elektrostatische Abstoßung die Agglomeration der Partikel. Die Folge ist ein Haufwerk mit einer dichten Struktur und einer homogenen Porengrößenverteilung. Ungeladene Partikel bilden hingegen große Agglomerate aus. Dies führt $\mathrm{zu}$ einer lockeren Haufwerksstruktur mit großen Poren, in denen der größte Teil des Flüssigkeitstransports stattfindet. Diesen Einfluss der Haufwerksstruktur auf die Durchströmbarkeit bezeichnet man als elektrostatischen Effekt [2].

- Zwischen der Flüssigkeit und den Partikeln wirkt die hydrodynamische Widerstandskraft. Bei makroskaligen Partikeln kann man den druckgetriebenen Durchfluss mit der Gleichung von Darcy [3] berechnen. Bei nanoskaligen Partikeln sind jedoch zusätzliche elektrokinetische Effekte zu berücksichtigen. Die strömende Flüssigkeit schert die Gegenionen aus dem diffusen Teil der Doppelschicht ab, so dass es $\mathrm{zu}$ einem Ladungstransport, dem Strömungsstrom $I_{\text {str }}$ kommt. Der Ladungstransport wird durch einen Gegenstrom ausgeglichen, der auf einer Ionenwanderung entgegen der Strömung und auf einer Oberflächenleitfähigkeit der Partikel beruht. Das treibende Potenzial für diesen Gegenstrom ist das Strömungspotenzial $U_{\text {str }}$, das zwischen beiden Seiten des Haufwerkes messbar ist. Es lässt sich mit:

$$
U_{\text {str }}=\frac{\varepsilon_{0} \varepsilon \zeta \Delta p}{\eta\left(K_{\mathrm{L}}+2 K_{\sigma} / a\right)}
$$

\begin{tabular}{|l|c|c|c|}
\hline $\mathrm{mm}$ & $\begin{array}{c}\text { Partikel-Partikel- } \\
\text { Wechselwirkung }\end{array}$ & $\begin{array}{c}\text { Wechselwirkung } \\
\text { mit Strömung }\end{array}$ & Externe Kraftfelder \\
\hline$\mu \mathrm{m}$ & Kontaktkraft & Widerstandskraft & $\begin{array}{c}\text { Gravitationsfeld, } \\
\text { Zentrifugalfeld, } \\
\text { Druckgradient }\end{array}$ \\
\hline $\mathrm{nm}$ & $\begin{array}{c}\text { Van der Waals-, } \\
\text { Coulomb-, Born-Kraft }\end{array}$ & $\begin{array}{c}\text { Deformation der EDL, } \\
\text { Strömungspotenzial }\end{array}$ & Elektrisches Feld \\
\hline
\end{tabular}

Tabelle 1. Übersicht der treibenden Kräfte und Wechselwirkungen bei der Durchströmung von Haufwerken verschiedener Größenskalen.

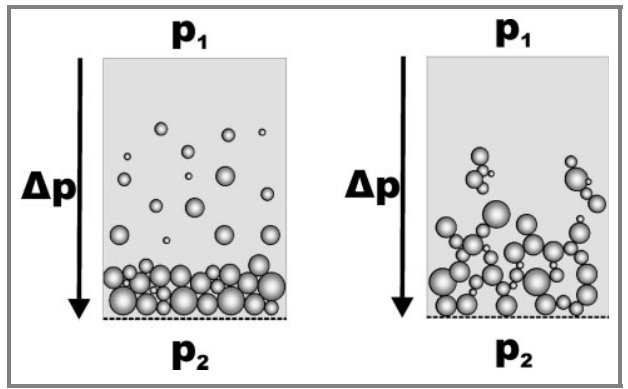

Abbildung 1. Ausbildung eines Haufwerkes mit lockerer Struktur durch Filtration einer agglomerierten Suspension am isoelektrischen Punkt (links), Ausbildung eines Haufwerkes mit dichter Struktur durch Filtration einer desagglomerierten Suspension mit hohem Zeta-Potenzial (rechts).

aus dem Zeta-Potenzial berechnen, wenn die Leitfähigkeit der Elektrolytlösung $K_{\mathrm{L}}$, die Oberflächenleitfähigkeit der Partikel $K_{\sigma}$ und der typische Krümmungsradius der Partikel $a$ bekannt sind [1]. Dabei ist $\varepsilon$ die relative Permittivität der Elektrolytlösung, $\varepsilon_{0}$ die Permittivität des Vakuums und $\eta$ die Viskosität der Flüssigkeit.

- Externe Kraftfelder, die üblicherweise die Durchströmung eines Haufwerkes bewirken, sind das Gravitationsfeld, ein Zentrifugalfeld oder ein Druckgradient. Die Durchströmung von nanoporösen Haufwerken ist jedoch durch den hohen Widerstand der kleinen Poren limitiert. Sie kann aber mit Hilfe von elektrischen Feldern, die die beweglichen Ionen im diffusen Teil der elektrochemischen Doppelschicht beschleunigen, verbessert werden. Die Ionen bewegen das sie umgebende Wasser aufgrund der viskosen Widerstandskraft durch die Poren. Dieser elektroosmotische Fluss $\dot{V}_{\text {eo }}$ ist mit:

$\dot{V}_{\mathrm{eo}}=-\frac{\varepsilon_{0} \varepsilon \zeta I}{\eta K_{\mathrm{L}}}$

eine Funktion der elektrischen Stromstärke $I$ [1]. Der elektroosmotische Volumenstrom erhöht oder vermindert die druckgetriebene Durchströmung eines Haufwerkes und kann auch dazu eingesetzt werden, Flüssigkeiten zu pumpen. Der gesamte Volumenstrom $\dot{V}$ ergibt sich als Summe der hydraulisch und elektroosmotisch getriebenen Durchströmung und ist in Anlehnung an Yeung and Mitchell [5] als lineare Funktion der Druckdifferenz $\Delta p$ und der Spannung $U$

$\dot{V}=C_{1}(-\Delta p)+C_{2}(-U)$

beschreibbar. Die Koeffizienten $C_{1}$ und $C_{2}$ sind hauptsächlich durch die Partikelgröße und das Partikelmaterial, die Kompression des Haufwerkes, den pH-Wert und die Ionenstärke der Elektrolytlösung in den Poren bestimmt. 


\section{Experimente}

Die Haufwerke wurden durch Filtration von wässrigen Suspensionen von Titandioxidpartikeln HT 1701 (TOHO Titanium, Japan) gebildet. Die Primärpartikel sind durch Sinterbrücken miteinander verbunden und bilden dadurch harte, nicht-sphärische Agglomerate. Die nach der BET-Methode ermittelte spezifische Oberfläche von $14,4 \mathrm{~m}^{2} / \mathrm{g}$ ergibt einen Sauter-Durchmesser von $105 \mathrm{~nm}$. Ein AcoustoSizer II (Colloidal Dynamics) diente zur Bestimmung des Zeta-Potenzials einer Suspension mit einem Feststoffmassenanteil von 20 \%. Das Zeta-Potenzial nimmt mit dem $\mathrm{pH}$ Wert monoton ab. Der isoelektrische Punkt liegt bei einem $\mathrm{pH}$-Wert von 6,5. Die Partikel haben eine Dichte von $4000 \mathrm{~kg} / \mathrm{m}^{3}$.

Die Suspensionen bestanden aus $30 \mathrm{~g}$ Titandioxid und $100 \mathrm{~g}$ Elektrolytlösung, deren $\mathrm{pH}$ Wert und Ionenkonzentration mit Salpetersäure, Kalilauge bzw. Kaliumnitrat eingestellt wurde. Eine Evakuierung bei $7 \mathrm{kPa}$ half, eingebrachte Luftblasen zu entfernen. Anschließend wurde die Suspension im Ultraschallbad dispergiert. Die Haufwerksbildung erfolgte durch beidseitige Filtration der Suspension in der Elektro-Kompressions-Permeabilitätszelle (EKP-Zelle), deren Querschnittsfläche $51,5 \mathrm{~cm}^{2}$ beträgt. Der über einen pneumatischen Stempel auf des Haufwerk aufgebracht Kompressionsdruck betrug $p_{\mathrm{c}}=2 \cdot 10^{5} \mathrm{~Pa}$. Mit einer Haufwerkshöhe von etwa $3 \mathrm{~mm}$ ergaben sich Porositäten von etwa $50 \%$.

Das große Verhältnis von Durchmesser zu Höhe des Haufwerkes verringert den Einfluss der Wandreibung auf die lokale Kompression. Nachdem der Filtratstrom abgeklungen und die Filtration beendet ist, beginnt die Durchströmung des Haufwerkes in der EKP-Zelle, die in Abb. 2 dargestellt ist. Die treibende Kraft ist dabei die Druckdifferenz zwischen beiden Seiten des Haufwerkes bzw. eine elektrische Spannung zwischen den Arbeitselektroden. Letztere sind mit Iridiumoxid beschichtet, um eine Oxidation $\mathrm{zu}$ verhindern. Die linke Arbeitselektrode ist als Anode, die rechte als Kathode festgelegt. Durch eine Spannungsumkehr lässt sich die Richtung der elektroosmotischen Strömung verändern.

Beim Ladungstransport entsteht an beiden Arbeitselektroden Elektrolysegas. Da die Gasblasen das Wasser im Ablaufsystem der EKPZelle verdrängen würden und somit als zusätzlicher Volumenstrom auf der Waage sichtbar wären, ist deren Entfernung mit Hilfe eines Spülsystems notwendig. Auf beiden Seiten des Haufwerkes werden die Arbeitselektroden mit der gleichen Elektrolytlösung umspült, die

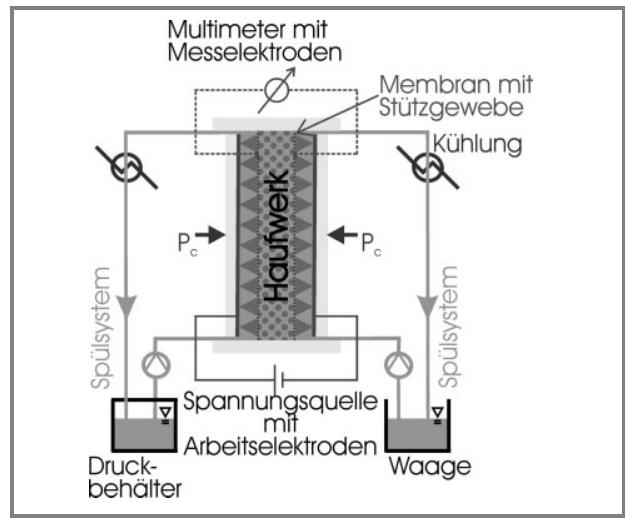

Abbildung 2. Prinzipskizze der Elektro-Kompressions-Permeabilitäts-Zelle.

auch das Haufwerk durchströmt. Die Kühlung der Spülkreisläufe hält die Temperatur des Haufwerkes trotz der bei Ladungstransport generierten Wärme konstant bei 288 K. Da ein Teil der Spannung wegen des elektrischen Widerstandes der Elektrolytlösung in den Spülsystemen verloren geht, befinden sich platinbeschichtete Messelektroden direkt an den Membranen. Diese Messelektroden ermöglichen die Messung der effektiven Spannung am Haufwerk, die einen elektroosmotischen Volumenstrom hervorruft bzw. als Strömungspotenzial erzeugt wird. Auch hier ist die linke Messelektrode die Anode, die rechte Messelektrode die Kathode.

Das Haufwerk wurde mit Elektrolytlösungen bei unterschiedlichen $\mathrm{pH}$-Werten und Ionenstärken durchströmt. Der $\mathrm{pH}$-Wert beeinflusst die Oberflächenladung der Partikel, während die Ionenstärke die Debye-Länge bestimmt. Die Einstellung des pH-Wertes von deionisiertem Wasser erfolgte durch Zugabe von Salpetersäure bzw. von Kalilauge. Kaliumund Nitrat-Ionen sind indifferente Ionen, die auf der Partikeloberfläche nicht spezifisch adsorbieren und deshalb die innere HelmholtzSchicht nicht beeinflussen. Bei der Untersuchung des Einflusses des pH-Wertes auf das Strömungspotenzial und den elektroosmotischen Volumenstrom lag die Ionenstärke der Elektrolytlösung bei $0,001 \mathrm{~mol} / \mathrm{L}$. Bei einem pH-Wert von 7 wurde die Ionenstärke variiert, um den Einfluss der Debye-Länge auf die elektrokinetischen Effekte zu untersuchen.

Vor den Messungen erfolgte eine mehrstündige druckgetriebene Durchströmung des Haufwerkes, bis die Elektrolytlösung in den Poren 30-mal ausgetauscht war. Die regelmäßige Kontrolle des Strömungspotenzials bestätigte das Erreichen einer konstanten Konzentration der Elektrolytlösung in den Poren. 


\section{Ergebnisse}

\subsection{Strömungspotenzial}

Das Strömungspotenzial wurde bei der druckgetriebenen Durchströmung gemessen. Dabei lag die Permeabilität des Haufwerkes $C_{1}$ bei etwa $5 \cdot 10^{-14} \mathrm{~m}^{3} /(\mathrm{Pa} \mathrm{s})$. Abb. 3 zeigt das Strömungspotenzial, das sich bei der druckgetriebenen Durchströmung des Haufwerkes mit Elektrolytlösungen verschiedener $\mathrm{pH}$ Werte ausbildet. Die Ionenstärke betrug 0,001 $\mathrm{mol} / \mathrm{L}$, so dass man von einer konstanten Debye-Länge ausgehen kann. Für jede verwendete Elektrolytlösung ist das Strömungspotenzial proportional zur Druckdifferenz. Entsprechend Gl. (1) wurde bei hohen $\mathrm{pH}$-Werten (9 und 11), also bei negativem Zeta-Potenzial, ein negatives Strömungspotenzial gemessen. Bei den pH-Werten 3, 5 und 7 ergab sich ein positives Strömungspotenzial, was auf ein positives Zeta-Potenzial hindeutet. Folglich liegt

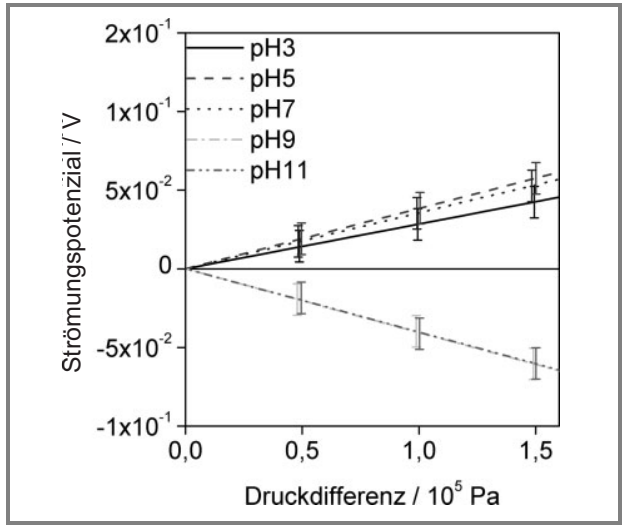

Abbildung 3. Strömungspotenzial als Funktion de Druckdifferenz. Das Haufwerk wurde von Elektrolytlösungen mit variierendem $\mathrm{pH}$-Wert und einer Ionenkonzentration von $0,001 \mathrm{~mol} / \mathrm{L}$ durchströmt.

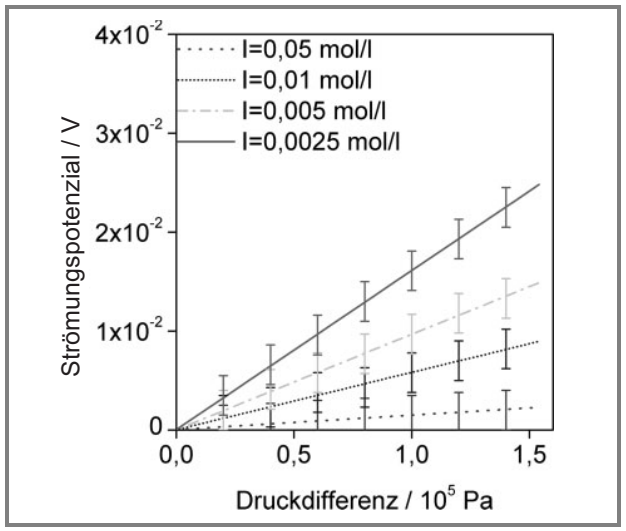

Abbildung 4. Strömungspotenzial als Funktion der Druckdifferenz. Das Haufwerk wurde von Elektrolytlösungen mit pH 7 und variierender lonenkonzentration durchströmt. der isoelektrische Punkt zwischen 7 und 9. Bei einem $\mathrm{pH}$-Wert von 3 war das Strömungspotenzial und damit das Zeta-Potenzial kleiner als bei einem $\mathrm{pH}$-Wert von 5 . Dies weicht von dem in der Suspension gemessenen, monotonen Verlauf des Zeta-Potenzials ab. Da das Zeta-Potenzial aber konzentrationsabhängig ist, kann man im Haufwerk nicht den gleichen Verlauf wie in der Suspension erwarten.

Abb. 4 zeigt das Strömungspotenzial in einem Haufwerk bei der Durchströmung mit Elektrolytlösungen verschiedener Ionenstärken bei einem $\mathrm{pH}$-Wert von 7. Für jede der Elektrolytlösungen ist das Strömungspotenzial proportional zur Druckdifferenz. Das positive Vorzeichen des Strömungspotenzials zeigt das positive Zeta-Potenzial der Partikel. Das Strömungspotenzial ist bei hoher Ionenstärke wesentlich kleiner als bei niedriger Ionenstärke. Anhand von Gl. (1) lässt sich dieses Verhalten erklären: Eine höhere Ionenstärke bedingt ein kleineres Zeta-Potenzial und eine höhere Leitfähigkeit der Elektrolytlösung. Die höhere Leitfähigkeit erleichtert den Ladungsrücktransport, der der Strömungsrichtung entgegengesetzt ist. Deshalb ist das Strömungspotenzial geringer, das für den Ausgleich des Strömungsstromes benötigt wird.

\subsection{Elektroosmose}

Die Anwendung eines elektrischen Feldes auf ein Haufwerk erzeugt einen elektroosmotischen Volumenstrom, wobei der Koeffizient der Elektroosmose $C_{2}$ je nach pH-Wert des Permeates zwischen $-2,8 \cdot 10^{-10} \mathrm{~m}^{3} /(\mathrm{V} \mathrm{s})$ und $1,7 \cdot 10^{-10} \mathrm{~m}^{3} /(\mathrm{V} \mathrm{s})$ liegt. In Abb. 5 ist die Fließrate für Elektrolytlösungen bei verschiedenen $\mathrm{pH}$-Werten dargestellt. Dabei handelt es sich um dieselben Elektrolytlösungen, die bei

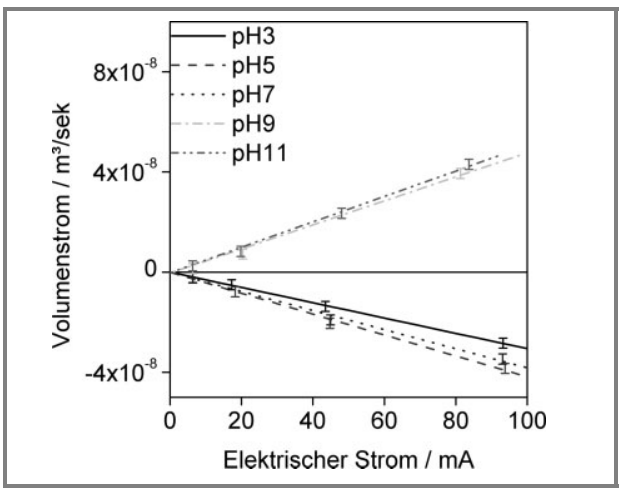

Abbildung 5. Elektroosmotischer Volumenstrom als Funktion der Stromstärke. Das Haufwerk wurde von Elektrolytlösungen mit variierendem $\mathrm{pH}$-Wert und einer lonenkonzentration von $0,001 \mathrm{~mol} / \mathrm{L}$ durchströmt. 


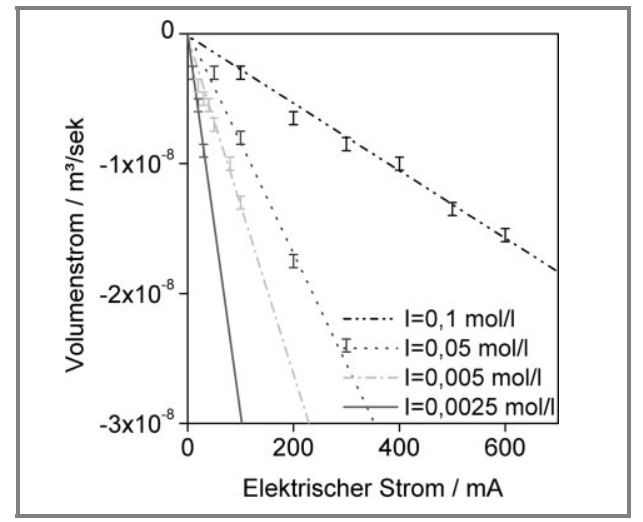

Abbildung 6. Elektroosmotischer Volumenstrom als Funktion der Stromstärke. Das Haufwerk wurde von Elektrolytlösungen mit $\mathrm{pH} 7$ und variierender lonenkonzentration durchströmt.

der Untersuchung des Strömungspotenzials verwendet wurden. Die Fließrate ist entsprechend Gl. (2) proportional zum elektrischen Strom. Positive Fließraten (Fließrichtung von links nach rechts in Abb. 2, also Zunahme der Masse auf der Waage) wurden bei positiver Richtung des elektrischen Feldes (Anode links, Kathode rechts) für die $\mathrm{pH}$-Werte 9 und 11 gemessen. Dies zeigt ein negatives Strömungspotenzial an. Bei den pH-Werten 3,5 und 7 traten bei positiver Richtung des elektrischen Feldes negative Fließraten auf. Dies ist ein Zeichen für ein positives Zeta-Potenzial. Bei pH 3 war die Fließrate kleiner als bei pH 5. Die Messung des elektroosmotischen Volumenstroms zeigt somit dieselbe Abhängigkeit des ZetaPotenzials vom $\mathrm{pH}$ Wert wie die Messung des Strömungspotenzials.

Abb. 6 zeigt den elektroosmotischen Volumenstrom bei pH 7 für verschiedene Ionenstärken. Das positive Zeta-Potenzial bedingt eine negative Fließrate, die bei hoher Ionenstärke wesentlich kleiner ist als bei niedriger Ionenstärke. Dies lässt sich folgendermaßen erklären: Bei niedriger Ionenstärke ist die Ausdehnung der elektrostatischen Doppelschichten, in denen die Gegenionen im Überschuss vorliegen, groß. Durch ein elektrisches Feld werden die Gegenionen beschleunigt, diese bewegen das sie umgebende Wasser, und es kommt zu einem elektroosmotischen Volumenstrom. Bei hoher Ionenstärke sind die elektrostatischen Doppelschichten dagegen dünner. Da außerhalb der Doppelschicht die Anzahl der Anionen und Kationen überein stimmt, werden Ionen in beiden Richtungen beschleunigt. Die Elektroosmose tritt also nur innerhalb der Doppelschicht auf und ist deshalb bei hoher Ionenstärke geringer ausgeprägt.

\section{Zusammenfassung}

Stoff- und Ladungstransport in nanoporösen Haufwerken sind eng miteinander verknüpft. Wenn eine Elektrolytlösung aufgrund einer Druckdifferenz durch die Poren strömt, bildet sich durch teilweises Abscheren der Ionen in den elektrochemischen Doppelschichten ein elektrisches Potenzial aus. Dieses Strömungspotenzial hängt von der ionischen Zusammensetzung der Elektrolytlösung ab. Bei basischen Permeaten ist es negativ, bei sauren und neutralen positiv. Je höher die Ionenstärke, desto kleiner ist das Strömungspotenzial. Durch Anlegen eines externen elektrischen Feldes wird eine elektroosmotische Durchströmung des Haufwerkes hervorgerufen, deren Fließrate proportional zum elektrischen Strom ist. Bei basischen Lösungen strömt der Elektrolyt in Richtung des Feldes, bei sauren oder neutralen Lösungen ist die Fließrichtung dem elektrischen Feld entgegengesetzt. Eine höhere Ionenstärke führt $\mathrm{zu}$ einer niedrigeren Fließgeschwindigkeit.

Der Deutschen Forschungsgemeinschaft sei an dieser Stelle für die finanzielle Unterstützung im Rahmen des Schwerpunktprogramms 1164 Nano-und Mikrofluidik (NI 414/1-2) herzlich gedankt.

Eingegangen am 18. Juli 2006

\section{Formelzeichen}

$\begin{array}{lll}E & {[\mathrm{~V} / \mathrm{m}] \quad \text { elektrisches Feld }}\end{array}$

I $[\mathrm{A}] \quad$ elektrischer Strom

$K_{\mathrm{L}} \quad[\mathrm{mS} / \mathrm{m}] \quad$ Leitfähigkeit der Elektrolyt-

$K_{\sigma} \quad[\mathrm{S}] \quad$ Leitfähigkeit der Partikel-

$$
\text { oberfläche }
$$

$C_{1}, C_{2}[-]$

$p_{\mathrm{c}} \quad[\mathrm{Pa}]$

$U_{\text {st }} \quad[\mathrm{V}]$

$\dot{V} \quad\left[\mathrm{~m}^{3} / \mathrm{s}\right]$

$\dot{V}_{\text {eo }} \quad\left[\mathrm{m}^{3} / \mathrm{s}\right]$

a $\quad[\mathrm{m}]$

$\Delta p \quad[\mathrm{~Pa}]$

$\varepsilon \quad[-]$

materialspezifische Kons-

tanten

Kompressionsdruck

Strömungspotenzial

volumetrische Fließrate

volumetrische Fließrate aufgrund der Elektroosmose

typischer Kurvenradius der Partikel

Druckdifferenz

relative Permittivitât der

Elektrolytlösung

$\varepsilon_{0} \quad[$ As/V m $] \quad$ Permittivität des Vakuums

$\eta \quad[\mathrm{Pa} \mathrm{s}] \quad$ Viskosität

$\zeta \quad[\mathrm{V}] \quad$ Zeta-Potenzial

\section{Stoff- und Ladungs- transport in nano- porösen Haufwer- ken sind eng mit- einander verknüpft.}


Dipl.-Ing. B. Schäfer

(bastian.schaefer@mvm.uka.de),

Prof. Dr.-Ing. H. Nirschl,

Institut für Mechanische Verfahrenstechnik und Mechanik, Universität Karlsruhe (TH),

Straße am Forum 8, D-76131 Karlsruhe,

Germany.

\section{Literatur}

[1] J. Lyklema, Fundamentals of Interface and Colloid Science, Volume II: Solid-Liquid Interfaces, Academic Press Limited, London 1995.

[2] H. Nirschl, B. Schaefer, Chem. Eng. Technol. 2005, 28 (8), 862. DOI: 10.1002/ceat.200500079

[3] H. Darcy, Les fontaines publiques de la ville de Dijon, Dalmont, Paris 1856.

[4] H. Nirschl, R. Wengeler, B. Schäfer, Nanofluidics in Particle Processing, Proc. 18th Annual Conference 2005, AFS American Filtration \& Separations Society, Atlanta, USA, April 10-13, 2005.

[5] A. T. Yeung, J. K. Mitchell, Geotechnique 1993, 43, 121. 